showing HER2 amplification or overexpression. They suggest that patients without HER2 amplification or overexpression are treated with the less toxic CMF regimen, whereas those with HER2 amplification or overexpression should receive anthracycline-containing regimens such as CEF.

Original article Pritchard KI et al. (2006) HER2 and responsiveness of breast cancer to adjuvant chemotherapy. N Engl J Med 354: 2103-2111

\section{Tumor vascularity predicts prognosis in adult, but not pediatric, astrocytomas}

Astrocytomas are the most common form of brain tumor in both adults and children, and are associated with a poor prognosis. A Turkish group has investigated whether tumor vascularity can predict prognosis in adults and children with this tumor type.

Birlik and colleagues enrolled 45 adults (aged 19-82 years) and 25 children (aged 1-16 years) with astrocytomas. They determined the vascularity of the tumors according to microvessel density, vascular grading and Chalkley counting. During a median follow-up of 12-24 months, 22 adults and 4 children died from disease progression. In univariate analysis, all three tumor vascularity parameters were significantly associated with postoperative survival in adults ( $P \leq 0.005$ for all). Higher-grade tumor histology in adults and older age in the overall group also correlated with reduced survival. In multivariate analysis, microvessel density was a significant independent prognostic indicator $(P=0.001)$; adults with microvessel density $\geq 70$ per $200 \times$ field had significantly shorter survival than those with lower microvessel densities $(P<0.001)$. By contrast, tumor histology and vascular parameters were not significantly associated with survival in children. The authors suggest that the observed differences between adults and children probably arise from biological variation in patients of different ages, relating to the ability of different tumors to induce angiogenesis.

The authors conclude that quantification of tumor vascularity may be useful in planning and monitoring antiangiogenic therapies for adult astrocytomas, but might not be of benefit in pediatric astrocytomas. This hypothesis needs confirming in further studies with longer follow-up in pediatric patients with high-grade astrocytomas.

Original article Birlik B et al. (2006) Tumour vascularity is of prognostic significance in adult, but not paediatric astrocytomas. Neuropathol Appl Neurobiol [doi: 10.1111/ j.1365-2990.2006.00763.x]

\section{5-Fluorouracil therapy is ineffective in mismatch-repair- deficient colorectal cancer}

Mismatch-repair (MMR) deficiency is present in approximately $15 \%$ of colorectal cancers. Although 5-fluorouracil has been shown to be effective against stage III colorectal cancer, in vitro and clinical retrospective studies suggest that MMR-deficient tumors might be resistant to this agent. Jover et al. carried out a prospective study to investigate the relationship between MMR deficiency and adjuvant 5-fluorouracil therapy in 754 patients with colorectal cancer, 66 of whom had MMRdeficient disease. Median follow-up was 728.5 days.

In patients with stage II or III colorectal cancer, adjuvant 5-fluorouracil was associated with better overall and disease-free survival in patients with MMR-competent tumors, but had no effect on these outcomes in patients with MMR-deficient tumors. MMR status had no effect on probability of survival in patients who received 5-fluorouracil. In patients who did not receive 5-fluorouracil, MMR-deficient disease was associated with a better probability of survival than MMR-competent disease, although this result did not reach statistical significance.

Given the lack of efficacy of 5-fluorouracil in MMR-deficient colorectal cancer, the authors recommend that patients with stage II disease should be treated with surgery alone. Trials of other chemotherapeutic agents, such as irinotecan, should be undertaken for patients with stage III disease. According to the authors, the molecular characteristics of tumors need to be taken into account in the design of adjuvant chemotherapy regimens for colorectal cancer, and the use of immunohistochemistry or microsatellite instability analysis should be routine in the design of such regimens.

Original article Jover R et al. (2006) Mismatch repair status in the prediction of benefit from adjuvant fluorouracil chemotherapy in colorectal cancer. Gut 55: 848-855 